\title{
Aquaporins: A Multidisciplinary Perspective on the Water Channel Proteins
}

Gokce Alp 1, [PhD]

ORCID: 0000-0001-8155-7377

I. Ipek Bosgelmez ${ }^{2},[\mathrm{MD}]$

ORCID: 0000-0003-2528-1227

Yesim Oztas ${ }^{3},[\mathrm{MD}]$

ORCID: 0000-0002-8638-9744

${ }^{1}$ Department of Chemical Engineering, Faculty of Engineering, Hacettepe University

${ }^{2}$ Department of Pharmaceutical Toxicology, Faculty of

Pharmacy, Erciyes University, Kayseri, Turkey

${ }^{3}$ Department of Medical Biochemistry, Faculty of

\section{nee A B S TRA C T Cen}

Aquaporins are unique water channel proteins located at cell membranes that possess high water permeability and high solute rejection. Their primary function is to maintain the osmotic balance of the cells via regulating the water transport. However, their discovery had also provided the scientists to understand the pathophysiology of some diseases. In fact, aquaporins are shown to be strongly related to cancer by taking part in several tumor-related processes such as cell migration, cell proliferation and cell adhesion. Other than their functions in human body, recently, aquaporins have started to be used in engineering biomimetic membranes, for different applications such as desalination. This review investigates the properties and functions of the aquaporins in a multidisciplinary point of view and demonstrates the recent developments in aquaporin-based research.

Keywords: Aquaporins, water channel proteins, water transport, cell membrane, biomimetic membranes

Corresponding Author: Gokce Alp, PhD

Department of Chemical Engineering, Faculty of Engineering, Hacettepe University, Ankara, Turkey

e-mail: gdilli@hacettepe.edu.tr

telephone: 03122977400

Received: 20 May 2020, Accepted: 23 June 2020,

Published online: 24 June 2020

\section{INTRODUCTION}

Water is essential for all molecular interactions required for anabolic and catabolic reactions vital to sustain life. The first critical paper on explaining the mechanism of cellular osmotic balance dates back to 1970 [1]. The pioneering studies of the Georghe Benga and Peter Agre groups have revealed a protein, later named as aquaporin 1 (AQP1) in erythrocytes. In 1986, Benga and colleagues from Romania, demonstrated for the first time the presence and location of a water channel by labeling experiments in the human erythrocyte membrane [2]. In 1988, Agre et al. from United States of America, 
first originally named this protein as CHIP 28 (channel integral membrane protein of molecular weight $28 \mathrm{kD}$ ) [3] with regard to its function in the linkage of the membrane skeleton to the lipid bilayer in RBC, then later in 1992 they reported CHIP as the water channel protein in erythrocytes [4]. A year later, Agre group suggested the name aquaporin-1 instead of CHIP28 [5]. Agre received the Nobel Prize in Chemistry in 2003 for the discovery of the water channel proteins through the cell membrane [6].

Since then, the research on AQPs has emerged and has been adopted by many disciplines, some of which are presented in Figure 1. As depicted in this figure, the distribution of "Web of Science Categories" within the published items on the topic "Aquaporin" reveals 13640 records (as of 05.05.2020), and the top-5 topics are in the order of "Neurosciences," "Biochemistry Molecular Biology," "Clinical Neurology", "Cell Biology," and "Physiology" which also underlines the importance of these proteins in both research and clinical applications.

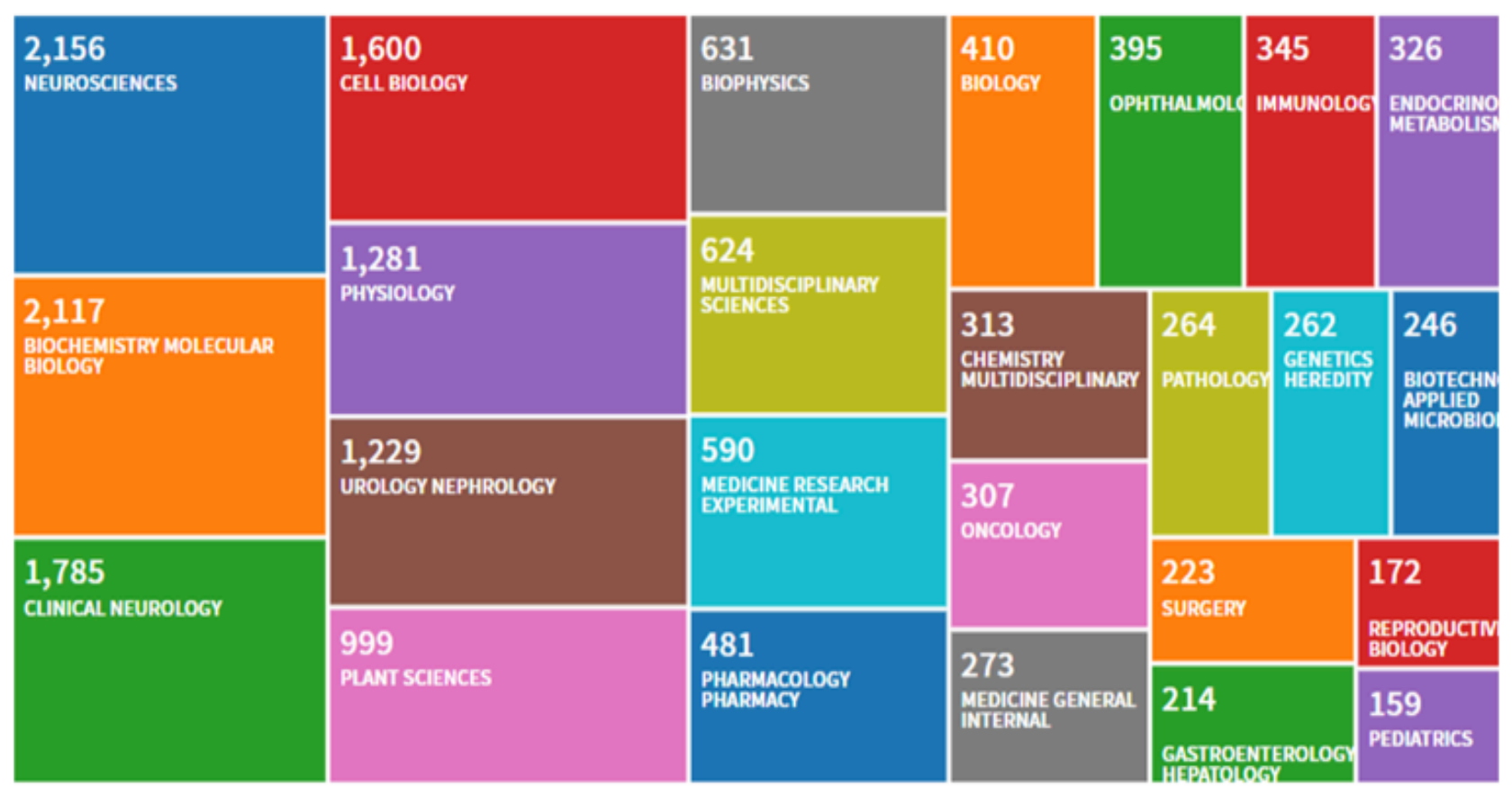

Figure 1. Distribution of top-25 "Web of Science Categories" within the published items related with the term "aquaporin" as of 05.05.2020

Regulation of the water transport is vital since water is the major component of living organisms and it is needed for several physiological processes.

In mammalians 13 isoforms of aquaporins (i.e. AQPO$A Q P 12)$ have been discovered to date and they all take part in different yet critical functions in diverse tissues. The numerous processes include concentration of urine in the kidney, fluid balance, secretion in the secretory glands, hydration of the skin, fertility in male, etc. As a natural result of the diversity of their functions, several research studies involving aquaporins have emerged.

In this context, there is increasing evidence that impairment of AQP function is associated with many diseases and pathological conditions and that studies on its regulation may be promising [7]. AQPs in mammals can be grouped into three main families: - AQPs with water permeability (AQP0, AQP1, AQP2, AQP4, AQP5, AQP6, AQP8),

- Aquaglyceroporins (AQP3, AQP7, AQP9, AQP10) which are permeable to both water and small molecules such as urea and glycerol.

- Those with suggested permeability to water (AQP 11, AQP 12) and glycerol (AQP 11).

In addition, it is stated that some of the AQPs are permeable to hydrogen peroxide $\left(\mathrm{H}_{2} \mathrm{O} 2\right)$ such as (AQP1, AQP3, AQP5, AQP8 and AQP9) and/or ammonia such as (AQP1, AQP3, AQP4, AQP6, AQP7, AQP8 and AQP9) [8].

Although water channel AQPs exhibit $\mathrm{H}_{2} \mathrm{O} 2$ permeability, permeability varies between isoforms; 
in addition, it is known that some $A Q P s$ may allow the passage of CO2 (AQP1, AQP4, AQP5), NO (AQP1, $A Q P 4)$ or $\mathrm{O} 2$ (AQP1, AQP4) [9].

\section{Structure of AQPs}

Aquaporins are hydrophobic membrane proteins with small sizes. Monomer sizes of the mammalian aquaporins are determined to be in the range of 26 to $34 \mathrm{kDa}$ [10]. Each monomer provides passage for a single water molecule. Several structural studies for analyzing the features of aquaporins are conducted via different methods such as epitope tagging, mutagenesis, freeze-fracture electron microscopy (EM) methods. From the freeze-fracture studies, it is shown that they are arranged as tetramers in the cell membrane [11]. As confirmed via various studies and cryo-EM images of mammalian erythrocyte
AQP1 and X-ray crystal structures of bovine AQP1, it has been suggested that aquaporins are in the form of an hourglass structure [12]. Each AQP monomer is composed from six a-helical domains, two half helices and five loops (Fig. 2) that originate a passage together by surrounding an amphipathic pore-like region with high selectivity $[7,10]$. As shown, carboxy- and amino-terminal domains are in the cytoplasm. At each monomer, three-amino-acid sequence (Asn-Pro-Ala) NPA motifs are present: one in the half helices located at the amino-terminal (M3) and one in the other half located at the carboxy-terminal (M7) [5]. These motifs contribute to the hydrophilic part of the amphiphilic pore and by acting as $\mathrm{H}$-bond donors/acceptors, they take part in coordinating water/glycerol transport through the aquaporin [13].

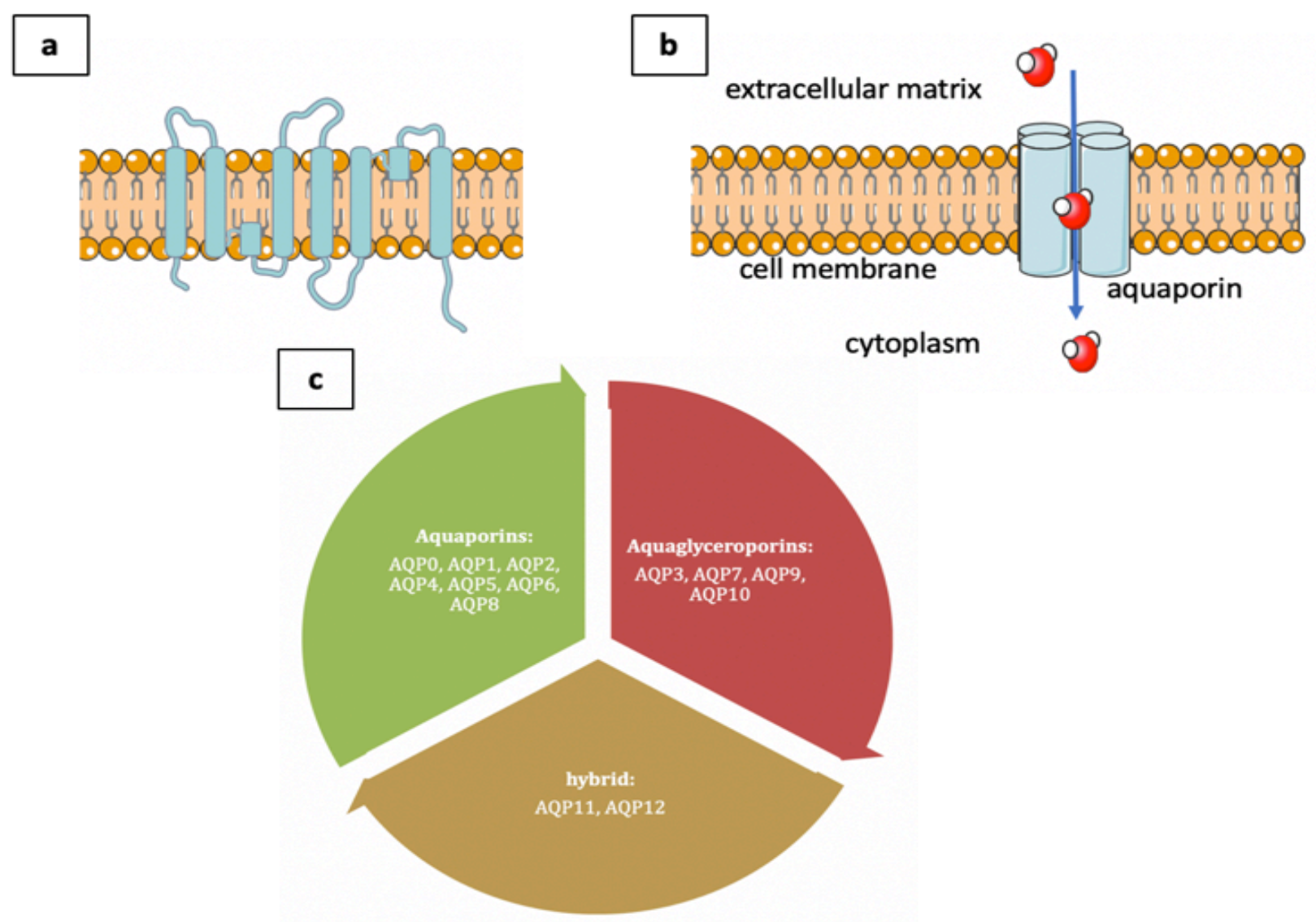

Figure 2. (a) Schematic representation of the helical domains, half domains and loops of a aquaporin monomer, (b) schematic representation of aquaporin water channel located in the cell membrane, (c) the aquaporin family in mammals. (This figure was drawn by using the image bank of Servier Medical Art. Servier Medical Art by Servier is licensed under a Creative Commons Attribution 3.0 Unported License. https://creativecommons.org/licenses/by/3.0/.) 


\section{Expression of AQPs}

Aquaporins are expressed in a variety of cells that are especially involved in fluid transport at plasma membranes such as epithelial cells along with the ones that do not possess a direct-function of fluid transport such as adipocytes [7].

Several AQPs are expressed in the eye including AQP0,

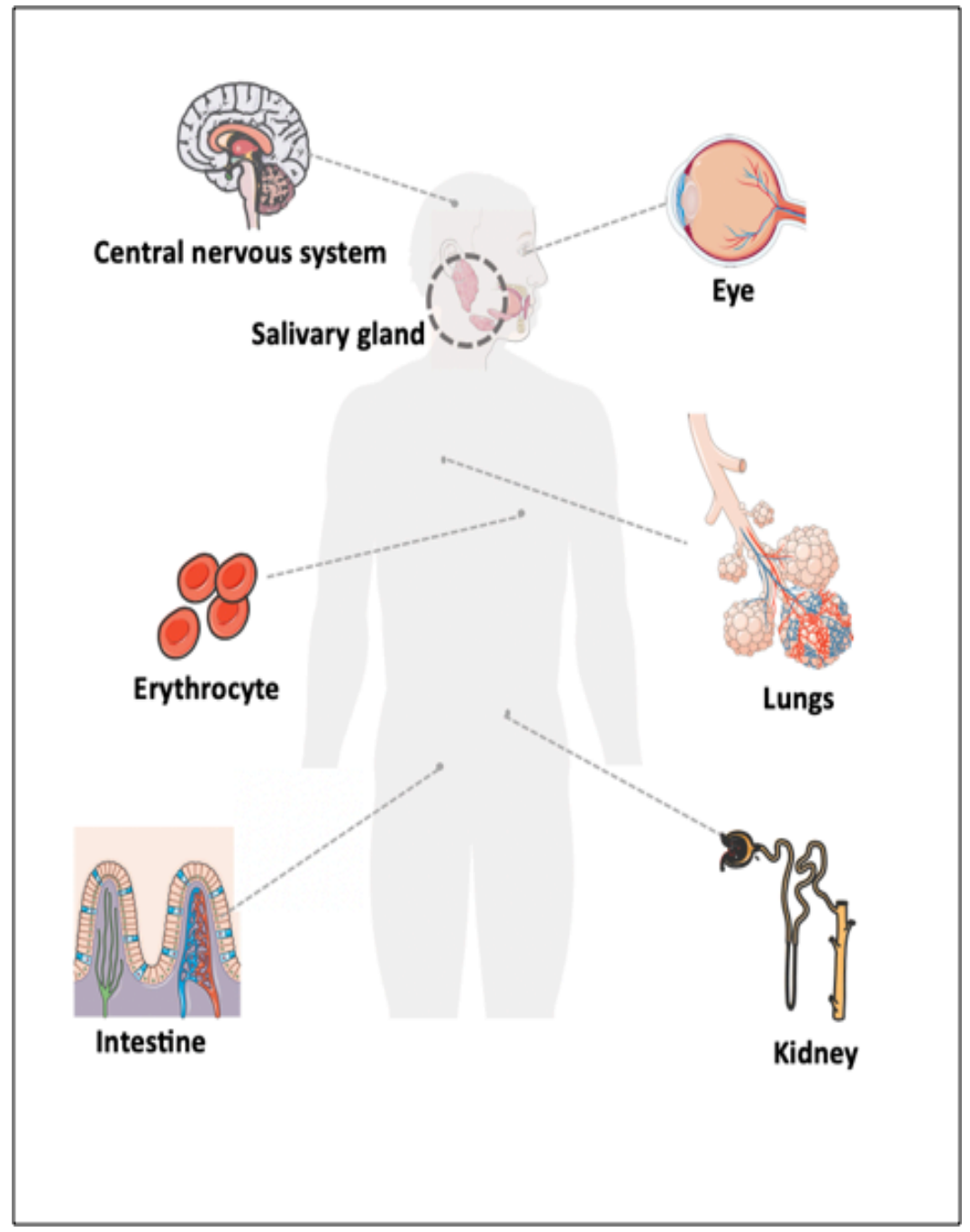

Figure 3. Distribution of mammalian aquaporins in human body. (This figure was drawn by using the image bank of Servier Medical Art. Servier Medical Art by Servier is licensed under a Creative Commons Attribution 3.0 Unported License. https://creativecommons.org/licenses/by/3.0/).

AQP1, AQP3, AQP4 and AQP5 which are expressed in the lens, corneal endothelium, conjunctiva, retinal cells and lacrimal gland, respectively [7]. In the central nervous system (CNS), AQP1 and AQP4 are expressed in choroid plexus, and astrocytes, respectively. In the submucosal glands of lung, AQP3 is expressed whereas in airways and alveolar type I cells, AQP4 and AQP5 are expressed. In the kidneys, numerous AQPs (2, 3, 4 and 7) are expressed and they take part in various functions. For example, AQP2 is expressed in collecting duct epithelial cells in response to presence of vasopressin and supports the increase the reabsorption of urine. AQP1, AQP3 and AQP9 are expressed in erythrocytes. AQP3, which is an aquaglyceroporin, is also expressed in the stratum corneum. Therefore, its deletion in mice causes dysfunctions in several processes such as wound healing or skin hydration as a result of inadequate amount of glycerol. Another aquaglyceroporin AQP7 is expressed in adipocytes which regulates glycerol transportation through the cell [14]. The tissue distributions of AQPs and their potential therapeutic effects are summarized in Table 1. 
Table 1. Tissue distribution of AQPs and their potential therapeutic effects (adapted from [7]).

\begin{tabular}{|c|c|c|c|}
\hline \multirow[b]{2}{*}{ AQP type } & \multirow[b]{2}{*}{ Tissues expressed in: } & \multicolumn{2}{|c|}{ Potential therapeutic effects } \\
\hline & & $\begin{array}{l}\text { Activation/ } \\
\text { upregulation }\end{array}$ & Inhibition \\
\hline AQPO & Lens & - & - \\
\hline AQP1 & $\begin{array}{l}\text { Renal tubules and microvessels, choroid plex- } \\
\text { us, ciliary epithelium, corneal endothelium, } \\
\text { pain-processing C-fibres, vascular endothelium } \\
\text { tumour vessels and red blood cells }\end{array}$ & - & $\begin{array}{l}\text { diuretic, reduced tumour angio- } \\
\text { genesis, reduced ocular pressure } \\
\text { in glaucoma }\end{array}$ \\
\hline AQP2 & Renal collecting duct & - & diuretic \\
\hline AQP3 & $\begin{array}{l}\text { Renal collecting duct, epidermis, conjunctiva, } \\
\text { corneal epithelium, immune cells, intestinal epi- } \\
\text { thelium and red blood cells }\end{array}$ & $\begin{array}{l}\text { enhanced skin hydration, en- } \\
\text { hanced wound healing }\end{array}$ & $\begin{array}{l}\text { diuretic, antitumour effects, } \\
\text { anti-inflammatory effects }\end{array}$ \\
\hline AQP4 & $\begin{array}{c}\text { Astrocytes, retinal Muller cells, } \\
\text { lacrimal gland, salivary duct, } \\
\text { inner ear, olfactory epithelium, gastric parietal } \\
\text { cells, airways, renal collecting duct, placenta, } \\
\text { muscle, gut epithelium and glioblastomas }\end{array}$ & $\begin{array}{l}\text { reduced vasogenic: CNS } \\
\text { oedema }\end{array}$ & $\begin{array}{c}\text { reduced cytotoxic CNS oedema, } \\
\text { anti-epileptic effects, promotion } \\
\text { of axonal regeneration, glioblas- } \\
\text { toma therapy }\end{array}$ \\
\hline AQP5 & $\begin{array}{l}\text { Corneal epithelium, sweat glands, lacrimal } \\
\text { glands, salivary glands, airway submucosal } \\
\text { glands, alveolar type I cells and epidermis }\end{array}$ & $\begin{array}{l}\text { restoration of salivary and lacri- } \\
\text { mal gland hypofunction }\end{array}$ & $\begin{array}{l}\text { reduced salvation and airway } \\
\text { mucus secretion }\end{array}$ \\
\hline AQP6 & $\begin{array}{c}\text { Intracellular vesicles in renal collecting duct in- } \\
\text { tercalated cells }\end{array}$ & - & - \\
\hline AQP7 & $\begin{array}{l}\text { Fat cells, renal proximal tubule, testis and } \\
\text { myocardium }\end{array}$ & anti-obesity effects & \\
\hline AQP8 & Intestinal epithelium & - & - \\
\hline AQP9 & $\begin{array}{l}\text { Hepatocytes, erythrocytes and possibly some } \\
\text { brain cells }\end{array}$ & - & - \\
\hline AQP10 & Intestinal enterochromaffin cells & - & - \\
\hline AQP11 & $\begin{array}{l}\text { Liver, testis and intracellular membranes in renal } \\
\text { proximal tubule }\end{array}$ & - & - \\
\hline AQP12 & Exocrine pancreas & - & - \\
\hline
\end{tabular}

\section{Functions of AQPs}

In mammalians, AQPs participate in many phys- glands (salivary/sweat), lung, kidney and intestine iological processes where secretion and absorp- [15]. Major physiological functions of AQPs related tion functions are required. These tissues include to their distribution are presented in Table 2.

Table 2. Major physiological functions of AQPs related to their locations

\begin{tabular}{|c|c|c|}
\hline & Location & Physiological Function/Outcome \\
\hline \multirow{5}{*}{ 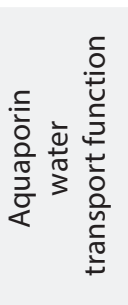 } & Kidney & Urine-concentration function \\
\hline & Epithelial fluid secretion & Secretion of saliva, CSF, aqueous humor \\
\hline & Cell migration & $\begin{array}{l}\text { Angiogenesis, tumor spread, wound healing, glial } \\
\text { scarring }\end{array}$ \\
\hline & Brain water transport & Brain swelling \\
\hline & Neuroexcitation & Neurosensation, seizure termination \\
\hline \multirow{3}{*}{ 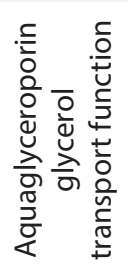 } & Skin hydration & Maintenance of epidermal glycerol \\
\hline & Cell proliferation & Tumor cell growth, wound healing \\
\hline & Adipocyte metabolism & Limits fat accumulation \\
\hline
\end{tabular}


Generally, AQPs maintain water flow through cell membranes by means of osmotic gradients occurring due to salt transport. Therefore, they play a vital role in the whole organism by providing a constant water homeostasis. In addition, they also play important roles in other cellular processes such as volume regulation, protein expression and cell adhesion [16].

In kidneys, AQP1, AQP2, AQP3 and AQP4 are the water channel proteins which are responsible for water absorption and elimination [14, 17]. In a study, Förster resonance energy transfer (FRET) measured by fluorescence lifetime imaging (FLIM-FRET) techniques has revealed a possible function of AQP1 in the $\mathrm{CO} 2$ transport and respiration [18].

In the central nervous system (CNS) AQP1 is essential for cerebrospinal fluid production along with the AQP4, which is important for edema fluid accumulation and elimination [14]. AQP5 is the one mainly found in apical plasma cell membrane of acinar epithelial cells in the salivary gland, and is responsible for osmotic water transport across the cells into the sweat duct [19].

Although AQPs are found in many tissues such as brain and kidney, their presence in erythrocytes is of particular importance. Erythrocytes are special cells that can reach even very narrow capillaries during their 120-day life in the circulation. Membrane flexibility and cellular elasticity, which allows these cells to pass easily through very thin capillaries, is critical to the movement of water into and out of the cell [20]. AQP1 is the most common type of water channel proteins, which was first shown in the cell membrane of erythrocytes in 1985. The first identified protein among the AQP was named as CHIP28, based on the fact that its molecular weight was 28$\mathrm{kDa}$, and then named as AQP1 as is used today [3, $11]$.

In addition to the pre-mentioned functions, recent studies report that AQPs also regulate other processes such as cell migration, cell proliferation and cell adhesion.

\section{Cell migration}

The unexpected cell migration ability of aquaporins was discovered in a study implemented by Loitto et. al., at which AQP9 in neutrophils were investigated [21]. In addition, it was demonstrated that a wide variety of AQPs including AQP1 and AQP4 which are expressed in aortic endothelial cells and astrocytes, respectively, assist cell migration in different cell types [22]. Although the mechanism is still unclear, it was concluded from different studies that AQP facilitate the migration via a chemotactic stimulus [23, 24]. It was reported that AQPs polarize at the leading side of a migrating cells so, it has been suggested that the presence of AQP enhances formation of the lamellipodium [25]. Moreover, it has been proposed that as the cells migrate through a tortuous extracellular space, the presence of AQP provide the spontaneous and fast changes in the shapes of the cells [26]. An "osmotic engine model" mechanism has been suggested for aquaporins to facilitate the changes in the cell shape. As changes in the cell volume can be obtained via water flow into and out of the cell, the role of AQPs is considered as important in cell migration [27].

\section{Cell proliferation}

It is known that AQP3 (expressed in the epidermis), improves the proliferation rate of basal keratinocytes. It has been found that wound healing is reduced in AQP3 null mice as a result of decrement at glycerol and ATP content in the keratinocytes. There is lack of information for the effect of AQP7 and AQP9 (aquaglyceroporins) on the proliferation. Also, there are some evidences that indicate that AQP3 and AQP5 play a role in tumor cell proliferation. However, further studies are still needed to gain a deeper understanding on the connection beneath AQP expression levels and tumor cell proliferation.

\section{Cell adhesion}

In the literature, recent studies have shown the relationship of AQPs, especially AQP0 or AQP4, with the cell adhesion. For example, it has been stated that AQP0, which is expressed in ocular system, takes part in cell-cell adhesion. In a study, implemented with AQPO null mice, it has been demonstrated that 
the presence of $A Q P 0$ is important to maintain the essential form of the lens with its integrity and transparency by providing the cell-cell adhesion [14]. In another study the role of AQP4 present in L-cells, a cell type lacking endogenous adhesion molecules, has been investigated and the presence of AQP4 in L-cells, which do not possess any adhesion molecules, has been suggested to cause the clustering of the cells, that has been explained through the structure of AQP4 which maintains weak interactions with other AQP4 proteins present in plasma cell membranes, thus helps to bind adjacent cells to one-another [28].

Lately, different studies revealed the possible relationship between AQPs and cancer [29]. It has been suggested that tumor growth and spread may be facilitated by the AQP expression in tumor cells and surrounding vessel [30, 31].

Recently, Maltaneri et al. have evaluated the relationship between AQP-1 and erythroid growth factor erythropoietin (Epo), which is proposed as an angiogenesis promoter. This study has shown that Epo may induce the expression of AQP1 at mRNA and protein levels, and inhibition of AQP1 can lead to an impairment in the migration of EA.hy 926 endothelial cells exposed to Epo, suggesting a potential use of aquaporin-targeting in angiogenesis-related diseases [32]. However, more specific target-oriented additional research is warranted to evaluate the possible beneficial role(s) of aquaporins in these areas.

\section{AQPs as Biomarkers}

In view of their roles in diverse cellular processes, various studies have focused on the possible use of AQPs as biomarkers. In this context, many studies have been published, most of which are related with cancer. For example, it has been reported that in pancreatic ductal adenocarcinoma (PDA) overexpression AQP3 at the plasma membrane of ductal cells has been reinforced in late and more aggressive PDA stages, while AQP5 expression has been related with tumor differentiation, which may be of use as a novel marker for PDA aggressiveness and intestinal infiltration [33]. Another more recent study proposed both AQP1 and AQP3 as diagnostic markers of PDA and a predictive marker of poor prognosis in PDA patients, in view of their findings that $A Q P 1$ and $A Q P 3$ expressions are associated with the tumorigenesis and progression of PDA. The area under the curve values of receiver operating characteristic for AQP1 and AQP3 have been reported as 0.669 and 0.707 , respectively [34]. A mechanistic study using large-scale proteomic analysis has revealed the significant relationship between AQP3 and the activity of mTOR signaling, suggesting that via activation of the mTOR signaling pathway AQP3 may promote tumor growth of pancreatic cancer cells; therefore this may provide a potential therapeutic target in the treatment [35].

In cervical cancer, it has been suggested that decreased expression of AQP1 correlated with progressive features in patients, and that AQP1 levels may serve as a potential biomarker for the diagnosis [36]. Both in prostate cancer cell lines and in benign and malignant human prostate tissue, the expression pattern, localization and potential clinical significance of 13 members of the AQP family have been probed, and the expression profile differences of several AQPs in benign and malignant prostate tissue have been shown. Expressions of AQP3, AQP4, AQP5, AQP7 and AQP9 have been confirmed using immunofluorescence microscopy; specifically, AQP3 protein expression heterogeneity in cancer specimens has been documented via immunohistochemistry studies, and the correlation between AQP3 expression and tumor grade has been underlined which may serve as a prognostic marker [37]. AQPs may also serve as viable biomarkers for renal diseases. A urine exosome study, in which excretion of AQP5 and AQP2 (UAQP5 and UAQP2), in 35 diabetic patients has shown that UAQP5 has been significantly increased in patients with diabetic nephropathy. In addition, UAQP5 and the histological class of diabetic nephropathy has been found to correlate positively. AQP2 has also been shown to reveal comparable results. Therefore, UAQP5 and UAQP2 have been proposed as noninvasive biomarkers in the diagnosis of diabetic nephropathy [38]. 


\section{AQPs as Treatment Strategies and Drug Targets}

The potential medical applications of AQP inhibitors as candidates in treatment of some diseases including disorders of central nervous system, eye, lung, as well as metabolic diseases and cancer have been reviewed previously [39, 40].

For example, AQP1 inhibition has been proposed as a novel strategy in anti-cancer therapy in a mouse model of melanoma [41]. Similarly, an endometriosis model in mice has shown that the silencing of AQP1 gene may activate the Wnt signaling pathway, that inhibit ectopic endometrial cell adhesion and invasion, suppress angiogenesis and promote apoptosis. In view of these findings, researchers have suggested that this approach may offer a new target that provide an improvement for the survival of patients with endometriosis [42].

Targeted studies on the potential compounds as modulators of expression or function of aquaporin/aquaglyceroporin have become an emerging research area. In this context, several approaches have been probed including gold(III) compound complexes [43], mercury compounds [44], as well as selective and potent isoform-specific aquaglyceroporin inhibitors [45].

A very recent study has applied a virtual screening method -molecular docking- to select potential compounds that bind to the AQP3, that is overexpressed in human skin squamous cell carcinoma, to be evaluated for the development of inhibitors to alleviate skin tumorigenesis [46].

It has been shown that dexamethasone and ambroxol can stimulate the mRNA and protein expression of AQP3 and AQP5 in a human airway epithelial cell line (A549 cells) which may imply the potential roles of these AQPs in the regulation of airway hypersecretion, as a target for treating related diseases [47]. In a more recent study, AQP5 has been detected in cell membrane and cytoplasm in human nasal epithelial cells, and methacholine intervention has inhibited expression of AQP5 while dexamethasone has been shown to increase the protein level significantly in a dose-dependent manner [48].

A study has highlighted a functionally critical increase in the nt-937 APQ5 promoter methylation linked to the binding of the inflammatorily acting nuclear transcription factor NF-KB in sepsis non-survivors, an it has been suggested that altering methylation at the $\mathrm{CpG}$-site nt-937 in the AQP5 promoter may constitute a potential therapeutic target in these patients [49].

In the case of metabolic disorders, such as obesity, diabetes and liver diseases, Calamita et al. have reviewed the possible role of AQPs. In addition, recent advances and challenges with respect to pharmacological modulation of the expression and function of AQPs to control and treat metabolic diseases have been discussed in detail [40] The role of and emerging points with regard to aquaglyceroporins in metabolic syndrome was discussed in a detailed review by Da Silva et al. [50]. The possible functions of aquaglyceroporins in adipose tissue may also provide a relevant field for the design of effective therapeutic compounds. It has been proposed that impaired glycerol transport through AQP7 has been correlated with triglyceride accumulation and obesity onset; moreover, some other isoforms, such as AQP3, AQP9, AQP10 and AQP11 have been identified in human adipocytes, although their involvement in the obesity mechanisms remain to be elucidated [51].

Last but not least, in a recent study, silybin has been found to restore the levels of AQP9 and glycerol permeability in an experimental hepatoma cell model of non-alcoholic fatty liver disease progression, besides other beneficial effects on hepatic steatosis. An interesting point underlined by the authors appears as the inverse association between hepatocyte AQP9 levels and autophagy accompanying triglyceride accumulation, restored via increased AQP9 expression [52].

On the other hand, it has been stated that despite considerable efforts to identify small molecule inhibitors of aquaporins, the activity of potential inhibitors could not be confirmed on re-testing possibly due to several reasons [53] underlining the importance of detailed studies. 


\section{Industrial and Future Applications of the Aquaporins through Biomimicry}

Other than their vital functions in cell membrane, $A Q P$ is expected to be useful in different industrial applications via establishing analogy with nature, i.e. through biomimicry. As they are known as protein channels facilitating water flow across cell membranes as a result of osmotic gradients, they can be used in various types of applications [14].

Simulating the function of aquaporins and applying it to different applications such as sensing, delivery processes and separation, depends mainly on understanding the dynamics of the transport through protein channels in cell membranes [54].

AQPs are in the shape of hourglass and during water transportation through the protein channel, each water molecule forms hydrogen bond with the inner-wall of the channel and also with an adjacent water molecule. Therefore, it is deduced that proton transport selectivity of AQPs is mainly controlled by the interactions between water molecules-pore and also by interactions of water molecules with one another. A channel-mediated transport mechanism is dominant in water transport through aquaporins. This provides an enhanced and fast water transport (囚108-109 water molecules/s.channel) combined with the rejection of all other solutes such as ions and protons [54]. Therefore, the idea of formation of AQP incorporated artificial membranes has emerged especially for desalination and water-purification applications $[55,56]$. If high water permeability and nearly perfect solute rejection can be maintained, an effective biomimetic membrane can be obtained. In cell membranes, main mechanism behind the water transportation is forward osmosis (FO) [57]. In humans, kidneys discharge waste and control the metabolite level via osmosis. AQPs are hydrophobic structures and it is known that they are able to self-assemble in phospholipids, so even an artificial kidney composed of AQP-incorporated biomimetic membrane may be designed if the lipids are chosen properly [58].

The idea of incorporating AQPs into the synthetic membranes was first hypothesized in the study by Kumar et al. in 2007, at which it was proposed that using AQP-integrated polymeric membranes provide high performance in terms of permeability $[55,59]$. Recently, AQP biomimetic membranes are preferred mainly in desalination applications using mostly Escherichia coli AqpZ. It has been postulated that permeability of AqpZ integrated biomimetic membranes would be about two orders of magnitude higher than the permeability of commercial reverse osmosis membranes [60]. Following these reports, various studies have been implemented on development of AQP biomimetic membranes and outstanding desalination performances have been obtained $[61,62]$. In earlier studies, AQPincorporated liposomes (proteoliposomes) or AQPincorporated supported lipid bilayer (SLB) membranes were formed via a facile and straightforward approach: vesicle rupture method. This method involves collapsing the proteoliposomes directly onto surface of a porous substrate, thus forming the skin layer $[59,61,63]$. Although this approach had been an effective example of biomimicry of the cell membrane and had attracted the attention of several scientists, low stability of the membrane had been a serious disadvantage among the preparation of a well-functioning biomimetic membrane. As the lipid based-skin layer is very thin (i.e. 4-8 nm), formation of a defect-free selective layer had been a real challenge. To overcome this challenge, a new procedure had been developed for fabrication of stable and mechanically strong membranes, in which AQP-incorporated vesicles were immobilized in a dense polymer layer. It was reported that membranes prepared with this method had exhibited more resistance to defect formation, thus provided satisfactory stability with good separation performance $[59,62]$. Also, to eliminate the drawbacks of SLB biomimetic membranes, an electrostatic layer-by-layer (LbL) assembly method was suggested as a new procedure for the production of robust membranes $[62,64]$. Recently, in a study implemented by Wang et al., AqpZ-incorporated SLB biomimetic membranes was fabricated via a novel LbL assembly method and as a result, it was reported that LbL method led to robust membranes with excellent separation performances [62]. 
Nowadays, removal of trace organics (such as pesticides) from water is one of the important procedures, which is still needed to be developed further. Existing FO membranes are reported to be ineffective against small neutral organic pollutants. Interestingly, in a study utilized by Madsen et al., a newly developed AQP integrated biomimetic membrane was used to test the removal of three selected pesticides which are known as important groundwater pollutants, namely: atrazine, 2,6-dichlorobenzamide (BAM) and desethyl-desisopropyl-atrazine (DEIA). It was stated that AQP integrated membranes possessed higher pure water flux than the commercial cellulose acetate membrane. Moreover, AQP membrane had managed to reject $\geq 97 \%$ of all trace organics, which is again a significantly higher value than the cellulose acetate membranes. The main reason for this difference was reported to be due to the difference between the mechanism of rejection, i.e. steric hindrance for the cellulose acetate membrane which results in a size dependent rejection vs. diffusion of trace organics for the AQP membrane [65].

In another study, the performance of an osmotic membrane bioreactor was analyzed which was constructed with a novel biomimetic AQP FO membrane. Removal of 30 types of trace organic contaminants including some pharmaceuticals, and pesticides, has been evaluated and the authors have suggested that some compounds such as salicylic acid, ketoprofen, naproxen, metronidazole, ibuprofen, gemfibrozil, pentachlorophenol, DEET, and ametryn could be removed by more than $80 \%$ in the bioreactor; whereas less than $30 \%$ removal rates have been observed for several other contaminants, including clofibric acid, fenoprop, primidone, carbamazepine, and atrazine. When compared with conventional cellulose triacetate and polyamide thinfilm composite FO membranes, it has been especially indicated that AQP-incorporated membrane had exhibited lower salt permeability which provided less salinity build-up in bioreactor during operation. Therefore, with stable and excellent contaminant removal performance and biomimetic AQP membranes are appropriate to be also used in such applications [66].

Another interesting and innovative approach is using AQP FO membranes in the algae dewatering for production of biofuel. In algal biofuel production, harvesting and dewatering steps of algae is a very energy consuming process, accepted as the major bottleneck limiting the development of microalgae-based fuel industry [67]. Recently, usage of FO membrane separation technology has been emerged to reduce the overall cost of energy. In a study implemented by Munshi et al., a new AQP incorporated polyethersulfone (PES) FO membrane was developed and its performance for algae dewatering was investigated for the first time in the literature [68].

As stated, different studies have been done for fabricating biomimetic FO membranes using AQP. However, the performance of these kinds of membranes may decrease since AQPs are of protein structures and they can be easily denatured during different procedures which involve usage of chemicals such as membrane cleaning.

Fabrication and application of aquaporin-based biomimetic membranes for water treatments have been demonstrated [69]. Li et al. have reported that a novel AQP-based biomimetic membrane had showed a good resistance against the tested chemical agents along with a stable flux and high salt rejection [70]. Similarly, AQP-incorporated reverse osmosis membrane with enhanced mechanical strength has been efficiently applied on a real seawater secondary effluent collected from a desalination plant in Singapore [69].

\section{CONCLUSION}

For the last 40 years, discovery of the aquaporins has provided significant impact on understanding the mechanism that stimulates osmotic balance in \& out of the cell and also on understanding the pathophysiology of some diseases. Moreover, the link between the aquaporins and cancer had been revealed, proving that aquaporins take part in several tumor-related processes. In addition to their major roles in cell membrane and medicine-related 
applications, recently, different applications of AQPs are proposed via several studies including preparation of AQP-incorporated biomimetic membranes for water desalination. Owing to their unique structure providing high water permeability and high solute rejection, the usage of aquaporins are expected to keep its increment with the studies implementing multidisciplinary approach and biomimicry.

\section{CONFUCT OF INTEREST STATEMENT}

The authors declare that there is no conflict of interest. 
[1] Macey, R.L. and R.E.L. Farmer, Inhibition of water and solute permeability in human red cells. Biochimica et Biophysica Acta (BBA) - Biomembranes 1970; 211(1): 104-106.

[2] Benga, G., etal., WaterPermeability in Human-Erythrocytes - Identification of Membrane-Proteins Involved in Water Transport. European Journal of Cell Biology 1986; 41(2): 252-262.

[3] Denker, B.M., et al., Identification, purification, and partial characterization of a novel $\mathrm{Mr} 28,000$ integral membrane protein from erythrocytes and renal tubules. Journal of Biological Chemistry 1988; 263(30): 15634-42.

[4] Preston, G.M., et al., Appearance of Water Channels in Xenopus Oocytes Expressing Red Cell CHIP28 Protein. Science 1992; 256(5055): 385-387.

[5] Agre, P., et al., Aquaporin CHIP: the archetypal molecular water channel. American Journal of Physiology-Renal Physiology 1993; 265(4): F463-F476.

[6] Agre, P., Aquaporin Water Channels (Nobel Lecture). Angewandte Chemie International Edition, 2004; 43(33): 4278-4290.

[7] Verkman, A.S., M.O. Anderson, and M.C. Papadopoulos, Aquaporins: important but elusive drug targets. Nature Reviews Drug Discovery 2014; 13(4): 259-277.

[8] Tesse, A., et al., Aquaporins as Targets of Dietary Bioactive Phytocompounds. Frontiers in Molecular Biosciences 2018; 5(30): 1-13.

[9] Almasalmeh, A., et al., Structural determinants of the hydrogen peroxide permeability of aquaporins. The FEBS Journal 2014; 281(3): 647-656.

[10] Verkman, A.S. and A.K. Mitra, Structure and function of aquaporin water channels. American Journal of Physiology-Renal Physiology 2000; 278(1): F13-F28.

[11] King, L.S., D. Kozono, and P. Agre, From structure to disease: The evolving tale of aquaporin biology. Nature Reviews Molecular Cell Biology 2004; 5(9): 687-698.

[12] Sui, H., et al., Structural basis of water-specific transport through the AQP1 water channel. Nature 2001; 414(6866): 872-878.

[13] Murata, K., et al., Structural determinants of water permeation through aquaporin-1. Nature 2000; 407(6804): 599-605.

[14] Papadopoulos, M.C. and S. Saadoun, Key roles of aquaporins in tumor biology. Biochimica et Biophysica Acta (BBA) - Biomembranes 2015; 1848(10, Part B): 2576-2583.

[15] Pelagalli, A., et al., Aquaporins in Health and Disease: An Overview Focusing on the Gut of Different Species. International Journal of Molecular Sciences 2016; 17(8): 1213.

[16] Kitchen, P., et al., Beyond water homeostasis: Diverse functional roles of mammalian aquaporins. Biochimica et Biophysica Acta (BBA) - General Subjects 2015; 1850(12): 2410-2421.

[17] Noda, Y., et al., Aquaporins in kidney pathophysiology. Nature Reviews Nephrology 2010; 6(3): 168-178.

[18] $\mathrm{Hsu}, \mathrm{K}$., et al., Adaptable interaction between aquaporin-1 and band 3 reveals a potential role of water channel in blood CO(2) transport. FASEB journal : official publication of the Federation of American Societies for Experimental Biology 2017; 31(10): 4256-4264.

[19] Ma, T., et al., Defective Secretion of Saliva in Transgenic Mice Lacking Aquaporin-5 Water Channels. Journal of Biological Chemistry 1999; 274(29): 20071-20074.8

[20] Huisjes, R., et al., Squeezing for Life - Properties of Red Blood Cell Deformability. Frontiers in physiology 2018; 9: 656-656.

[21] Loitto, V.-M., et al., Neutrophil leukocyte motility requires directed water influx. Journal of Leukocyte Biology 2002; 71(2): 212-222.

[22] Saadoun, S., et al., Involvement of aquaporin-4 in astroglial cell migration and glial scar formation. Journal of Cell Science 2005; 118(24): 5691-5698.

[23] Auguste, K.I., et al., Greatly impaired migration of implanted aquaporin-4-deficient astroglial cells in mouse brain toward a site of injury. The FASEB Journal 2007; 21(1): 108-116.

[24] Ruiz-Ederra, J. and A.S. Verkman, Aquaporin-1-facilitated keratocyte migration in cell culture and in vivo corneal wound healing models. Experimental Eye Research 2009; 89(2): 159-165.

[25] Bisi, S., et al., Membrane and actin dynamics interplay at lamellipodia leading edge. Current Opinion in Cell Biology 2013; 25(5): 565-573.

[26] Papadopoulos, M.C., S. Saadoun, and A.S. Verkman, Aquaporins and cell migration. Pflügers Archiv - European Journal of Physiology 2008; 456(4): 693-700.

[27] Stroka, Kimberly M., et al., Water Permeation Drives Tumor Cell Migration in Confined Microenvironments. Cell 2014; 157(3): 611-623.

[28] Zhang, H. and A.S. Verkman, Evidence against Involvement of Aquaporin-4 in Cell-Cell Adhesion. Journal of Molecular Biology 2008; 382(5): 1136-1143.

[29] Kasa, P., et al., Aquaporins in female specific cancers. Gene 2019; 700: 60-64.

[30] Verkman, A.S., M. Hara-Chikuma, and M.C. Papadopoulos, Aquaporins-new players in cancer biology. Journal of Molecular Medicine 2008; 86(5): 523-529.

[31] Hu, J. and A.S. Verkman, Increased migration and metastatic potential of tumor cells expressing aquaporin water channels. The FASEB Journal 2006; 20(11): 1892-1894.

[32] Maltaneri, R.E., et al., Aquaporin-1 plays a key role in erythropoietin-induced endothelial cell migration. Biochimica et biophysica acta. Molecular cell research 2020; 1867(1): 118569.

[33] Direito, I., et al., Differential expression of aquaporin-3 and aquaporin-5 in pancreatic ductal adenocarcinoma. Journal of Surgical Oncology 2017; 115(8): 980-996.

[34] Zou, W., et al., AQP1 and AQP3 Expression are Associated With Severe Symptoms and Poor-prognosis of the Pancreatic Ductal Adenocarcinoma. Applied Immunohistochemistry \& Molecular Morphology 2019; 27(1): 40-47.

[35] Huang, X., L. Huang, and M. Shao, Aquaporin 3 
facilitates tumor growth in pancreatic cancer by modulating mTOR signaling. Biochemical and Biophysical Research Communications 2017; 486(4): 1097-1102.

[36] Wei, M., et al., Decreased expression of aquaporin 1 correlates with clinicopathological features of patients with cervical cancer. OncoTargets and therapy 2019; 12: 2843-2851.

[37] Bründl, J., et al., Expression, localisation and potential significance of aquaporins in benign and malignant human prostate tissue. BMC urology 2018; 18(1): 75-75.

[38] Rossi, L., et al., Urinary Excretion of Kidney Aquaporins as Possible Diagnostic Biomarker of Diabetic Nephropathy. Journal of diabetes research 2017; 2017: 4360357-4360357.

[39] Huber, V.J., M. Tsujita, and T. Nakada, Aquaporins in drug discovery and pharmacotherapy. Molecular Aspects of Medicine 2012; 33(5): 691-703.

[40] Calamita, G., J. Perret, and C. Delporte, Aquaglyceroporins: Drug Targets for Metabolic Diseases? Frontiers in physiology $2018 ; 9: 851-851$

[41] Nicchia, G.P., et al., Inhibition of aquaporin-1 dependent angiogenesis impairs tumour growth in a mouse model of melanoma. Journal of Molecular Medicine 2013; 91(5): 613-623.

[42] Shu, C., et al., Inhibitory effect of AQP1 silencing on adhesion and angiogenesis in ectopic endometrial cells of mice with endometriosis through activating the Wnt signaling pathway. Cell Cycle 2019; 18(17): 2026-2039.

[43] de Almeida, A., et al., The mechanism of aquaporin inhibition by gold compounds elucidated by biophysical and computational methods. Chemical Communications 2017; 53(27): 3830-3833.

[44] Spinello, A., et al., The inhibition of glycerol permeation through aquaglyceroporin-3 induced by mercury(II): A molecular dynamics study. Journal of Inorganic Biochemistry 2016; 160: 78-84.

[45] Sonntag, Y., et al., Identification and characterization of potent and selective aquaporin-3 and aquaporin-7 inhibitors. The Journal of biological chemistry 2019; 294(18): 7377-7387.

[46] Yadav, D.K., et al., Computational Modeling on Aquaporin-3 as Skin Cancer Target: A Virtual Screening Study. Frontiers in chemistry 2020; 8: 250-250.

[47] Ben, Y., et al., Upregulation of AQP3 and AQP5 induced by dexamethasone and ambroxol in A549 cells. Respiratory physiology \& neurobiology 2008; 161(2): 111-118.

[48] Chang, Y.-L., et al., Dexamethasone attenuates methacholine-mediated aquaporin 5 downregulation in human nasal epithelial cells via suppression of NF-KB activation. International Forum of Allergy \& Rhinology 2018; 8(1): 64-71.

[49] Rump, K., et al., DNA methylation of a NF-KB binding site in the aquaporin 5 promoter impacts on mortality in sepsis. Scientific Reports 2019; 9(1): 18511.

[50] da Silva, I.V., et al., Revisiting the metabolic syndrome: the emerging role of aquaglyceroporins. Cellular and Molecular Life Sciences 2018; 75(11): 1973-1988.

[51] Madeira, A., T.F. Moura, and G. Soveral, Aquaglyceroporins: implications in adipose biology and obesity. Cellular and Molecular Life Sciences 2015; 72(4): 759-771.

[52] Baldini, F., et al., Aquaporin-9 is involved in the lipid-lowering activity of the nutraceutical silybin on hepatocytes through modulation of autophagy and lipid droplets composition. Biochimica et biophysica acta. Molecular and cell biology of lipids 2020; 1865(3): 158586.

[53] Tradtrantip, L., et al., Aquaporin-Targeted Therapeutics: State-of-the-Field, in Aquaporins, B. Yang, Editor. 2017, Springer Netherlands: Dordrecht. 239-250.

[54] Barboiu, M., Artificial water channels - incipient innovative developments. Chemical Communications 2016; 52(33): 5657-5665.

[55] Kumar, M., et al., Highly permeable polymeric membranes based on the incorporation of the functional water channel protein Aquaporin Z. Proceedings of the National Academy of Sciences 2007; 104(52): 20719-20724.

[56] Shen, Y.-x., et al., Biomimetic membranes: A review. Journal of Membrane Science 2014; 454: 359-381.

[57] Farinas, J. and A.S. Verkman, Cell volume and plasma membrane osmotic water permeability in epithelial cell layers measured by interferometry. Biophysical Journal 1996; 71(6): 3511-3522.

[58] Jakowiecki, J., et al., Aquaporin-graphene interface: relevance to point-of-care device for renal cell carcinoma and desalination. Interface focus 2018; 8(3): 20170066-20170066.

[59] Li, X., et al., Nature gives the best solution for desalination: Aquaporin-based hollow fiber composite membrane with superior performance. Journal of Membrane Science 2015; 494: 68-77.

[60] Tang, C.Y., et al., Desalination by biomimetic aquaporin membranes: Review of status and prospects. Desalination 2013; 308: 34-40.

[61] Li, X., et al., Preparation of supported lipid membranes for aquaporin Z incorporation. Colloids and Surfaces B: Biointerfaces 2012; 94: 333-340.

[62] Wang, M., et al., Layer-by-Layer Assembly of Aquaporin Z-Incorporated Biomimetic Membranes for Water Purification. Environmental Science \& Technology 2015; 49(6): 3761-3768.

[63] Werber, J., C. Osuji, and M. Elimelech, Materials for Next-Generation Desalination and Water Purification Membranes. Nature Reviews Materials 2016; 1: 16018.

[64] Masuda, K., et al., Fabrication and Conductive Properties of Multilayered Ultrathin Films Designed by Layer-byLayer Assembly of Water-Soluble Fullerenes. Langmuir 2010; 26(16): 13472-13478.

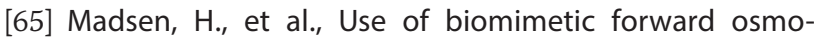
sis membrane for trace organics removal. Journal of Membrane Science 2015; 476: 469-474.

[66] Luo, W., et al., Biomimetic aquaporin membranes for osmotic membrane bioreactors: Membrane performance and contaminant removal. Bioresource Technology 2018; 249: 62-68.

[67] Uduman, N., et al., Marine microalgae flocculation and focused beam reflectance measurement. Chemical Engineering Journal 2010; 162: 935-940.

[68] Munshi, F., et al., Dewatering using an AquaporinBased Polyethersulfone Forward Osmosis Membrane. Separation and Purification Technology 2018; 204: 154-161.

[69] Li, Y., et al., Fabrication of aquaporin-based biomimetic membrane for seawater desalination. Desalination 2019; 467: 103-112.

[70] Li, Z., et al., Aquaporin based biomimetic membrane in forward osmosis: Chemical cleaning resistance and practical operation. Desalination 2017; 420: 208-215. 